\title{
Hematoma subdural agudo infratentorial: relato de caso
}

\author{
Leonardo Barnsley Araújo ${ }^{,}$, Raul Starling de Barros², Bruno Freire de Castro \\ Hospital de Pronto-Socorro João XXIII, Belo Horizonte, MG, Brasil.
}

\section{RESUMO}

Os autores relatam o caso de um paciente adulto vítima de traumatismo cranioencefálico e que apresentou uma lesão considerada rara na literatura, o hematoma subdural agudo da fossa posterior. O paciente foi submetido à craniectomia suboccipital com drenagem do hematoma e recebeu alta com escore de 3 pontos na Glasgow Outcome Scale; um bom resultado, pois essa lesão atinge $71 \%$ de mortalidade em algumas séries.

\section{PALAVRAS-CHAVE}

Traumatismos encefálicos, hematoma subdural agudo, fossa craniana posterior.

\section{ABSTRACT}

Posterior fossa acute subdural hematoma: case report

The authors report a case of acute subdural hematoma of posterior fossa in an adult male patient secondary to a head trauma. This is considered a rare pathology in literature. The patient was operated and discharged with a score 3 in GOS. This can be considered a good result since some series show a mortality of $71 \%$ for this pathology.

\section{KEYWORDS}

Brain injuries, acute subdural hematoma, posterior cranial fossa.

\section{Introdução}

Os autores relatam o caso de um paciente adulto com hematoma subdural agudo infratentorial (HSDA-I) unilateral, pós-traumático. Essa lesão é rara ${ }^{1}$ e contrasta com a maior incidência dos hematomas extradurais infratentoriais, comuns e de origem das fraturas occipitais e lesões do seio transverso. ${ }^{2}$

A incidência desse tipo de patologia, em algumas séries, é de apenas 3\% das lesões em todas as tomografias realizadas por trauma cranioencefálico (TCE); possivelmente essa é a patologia de menor prevalência e incidência nos TCEs. ${ }^{2-4}$

Deve-se ressaltar que o HSDA-I é mais frequente nas crianças $(0,52 \% \text { de todos os hematomas subdurais })^{2}$ e é geralmente desencadeado por trauma.
Trabalho de D’Avella et al. ${ }^{2}$ relata que até 1995 apenas 13 casos foram relatados na literatura.

Pacientes anticoagulados apresentam pior prognóstico, com $90 \%$ a $100 \%$ de taxas de morte.,

Os hematomas infratentoriais são lesões com alto índice de mortalidade, e a descompressão da fossa posterior é o tratamento de escolha e este deve ser realizado prontamente. $^{2-6}$

Pacientes com história de TCE, fratura occipital e sinais de compressão do tronco cerebral são aqueles que devem alertar para a suspeição de lesões da fossa posterior. $^{2}$

Conforme protocolo da clínica neurocirúrgica do Hospital de Pronto-Socorro João XXIII (HPS), a craniectomia descompressiva é o tratamento de escolha nos hematomas infratentoriais. ${ }^{5}$

Médico neurocirurgião do Hospital de Pronto-Socorro João XXIII, Belo Horizonte, MG, Brasil.

2 Médico neurocirurgião do Hospital de Pronto-Socorro João XXIII; médico preceptor do Instituto da Previdência dos Servidores do Estado de Minas Gerais, Belo Horizonte, MG, Brasil.

3 Médico-residente do Hospital da Baleia; médico estagiário do Hospital de Pronto-Socorro João XXIII, Belo Horizonte, MG, Brasil. 


\section{Caso clínico}

Paciente de 45 anos, masculino, lavrador, encaminhado de João Monlevade, cidade há 115 quilômetros de Belo Horizonte, vítima de atropelamento, e encontrado na cena com oito pontos na Escala de Coma de Glasgow (ECG).

No HPS, a TC revelou contusões temporais à esquerda (Figura 1) e HSDA-I à esquerda. Foi submetido à craniectomia suboccipital esquerda pelo neurocirurgião sênior do nosso serviço, RSB, com drenagem do hematoma (Figura 2). O córtex cerebelar parecia normal, sem contusões. Foi encaminhado ao CTI entubado, com PIC normal.

No sexto dia de pós-operatório apresentou aumento da PIC e das contusões temporais à esquerda. Foi então submetido à craniectomia descompressiva supratentorial à esquerda.

O paciente recebeu alta três meses após a internação, alerta, hemiparético direito, disfásico, com três pontos na Glasgow Outcome Scale.
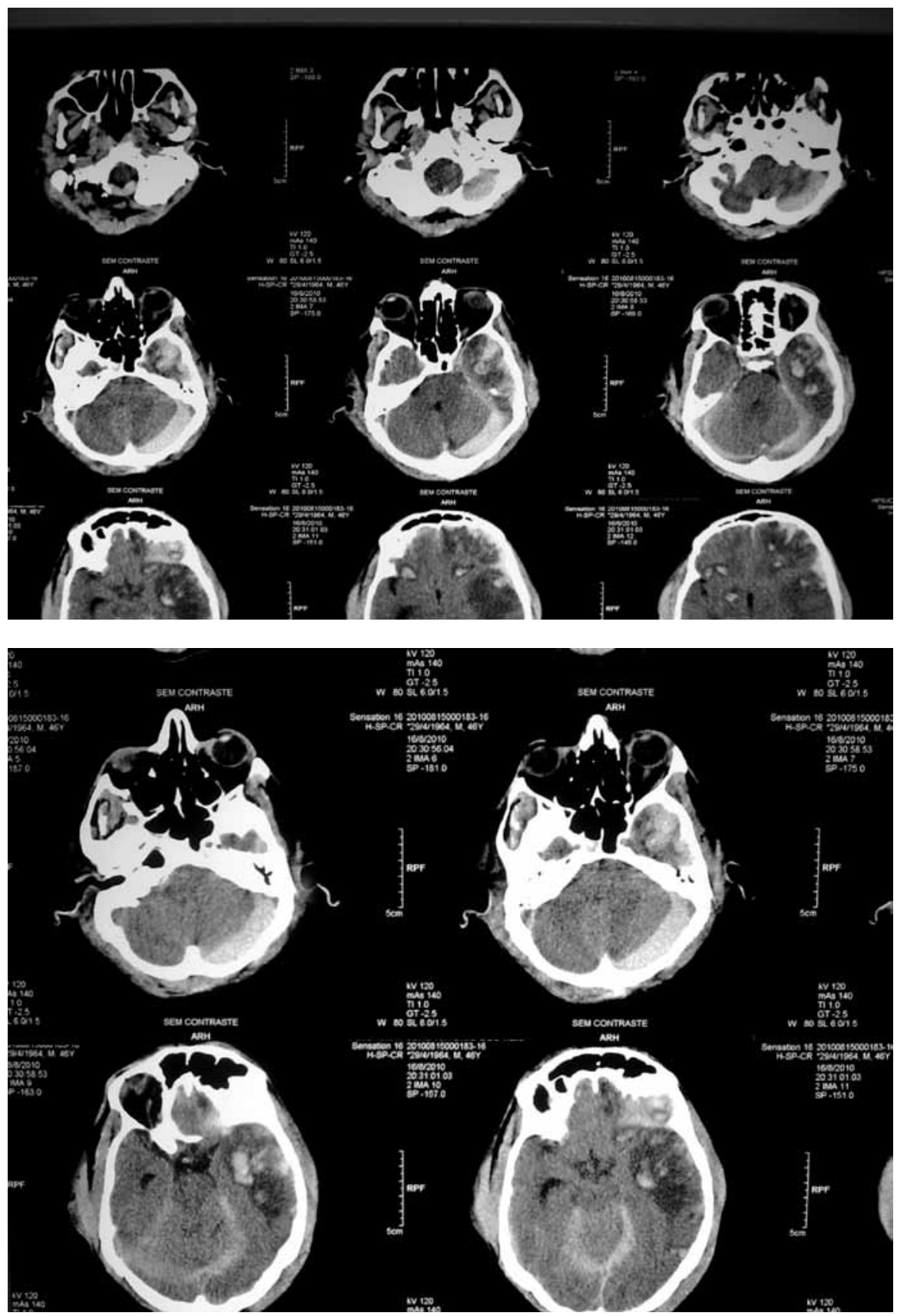

Figura 1 - Hematoma subdural agudo infratentorial e contusões múltiplas temporais do lado esquerdo. 


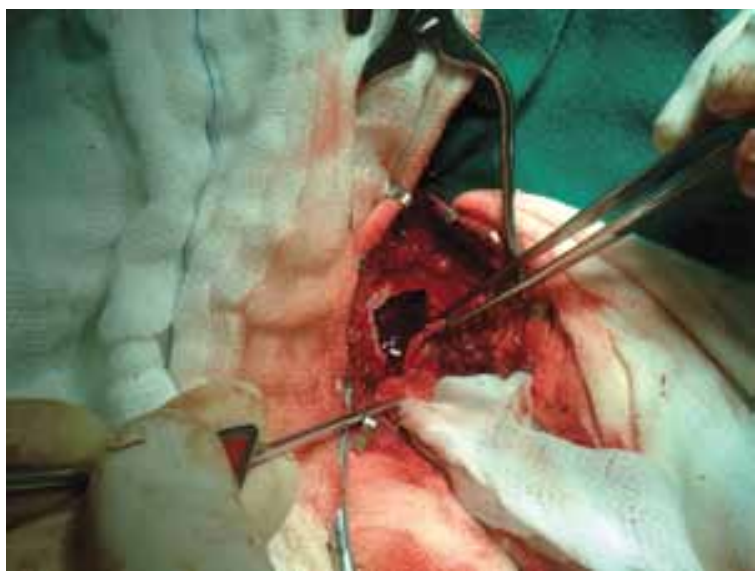

Figura 2 - Craniectomia suboccipital esquerda revelando extenso hematoma subdural agudo.

\section{Resultados e discussão}

Os hematomas infratentoriais com efeito de massa devem ser drenados. ${ }^{1-7}$

O principal fator prognóstico para os hematomas da fossa posterior é a condição clínica do paciente no momento da cirurgia, por isso essa condição deve ser prontamente tratada. ${ }^{1}$ Série de Seelig et al. ${ }^{8}$ com 20 pacientes comatosos e sinais de disfunção do tronco cerebral demonstrou que os sobreviventes foram operados aproximadamente 2,5 horas após o trauma, concluindo que o tempo para abordagem também é determinante no prognóstico.

Ashkenazi et al. ${ }^{9}$ relatam que uma abordagem conservadora pode ser instituída em pacientes com hematomas infratentoriais iguais ou menores de $1 \mathrm{~cm}$ e que não exerçam compressão sobre o tronco cerebral ou desloquem o quarto ventrículo; portanto, pacientes neurologicamente intactos e que possuam alterações radiográficas importantes devem ser operados.

Pacientes com HSDA-I e Glasgow abaixo de 8 possuem um prognóstico mais desfavorável no pós-operatório em relação àqueles pacientes com Glasgow acima de $8 .^{2}$

Motohashi et al. ${ }^{10}$ relatam o caso de dois pacientes com sinais de disfunção do tronco cerebral e hidrocefalia aguda, tratados com trepano-drenagens suboccipitais na sala de emergência, com resposta favorável. O nosso paciente, por apresentar quadro menos grave, propiciou a drenagem por craniectomia suboccipital clássica no bloco cirúrgico.
Algumas séries relatam até $100 \%$ de fratura occipital relacionada com os HSA-I. Contudo, no caso presente, a fratura estava do lado esquerdo, oposto ao do hematoma subdural agudo.

Embora a literatura relate até $71 \%$ de mortalidade para o HSDA-I, o paciente apresentou resultado satisfatório. Fez-se necessária uma segunda cirurgia apenas para o tratamento das contusões temporais à esquerda.

Por ser pouco comum, ainda não existe protocolo para o tratamento do HSDA-I, com referências ao volume, desvio de estruturas ou quadro clínico.

Os autores esperam que este artigo contribua para a conduta nessa patologia e estimule estudos com melhores níveis de evidência.

\section{Referências}

1. Young HA, Schmidek HH. Complications accompanying occipital skull fracture. J Trauma. 1982;22(11):914-20.

2. D'Avella D, Servadei F, Scerrati M, Tomei G, Brambilla G, Massaro F, et al. Traumatic acute subdural haematomas of the posterior fossa: clinicoradiological analysis of 24 patients. Acta Neurochir (Wien). 2003;145(12):1037-44.

3. Gulsen S, Sonmez E, Yilmaz C, Altinors N. Traumatic acute subdural hematoma extending from the posterior cranial fossa to the cerebellopontine angle. J Korean Neurosurg Soc. 2009;46(3):277-80.

4. Miranda P, Alday R, Lagares A, Pérez A, Lobato RD. Posterior fossa subdural hematoma mimicking intracerebellar hemorrhage. Neurocirugia (Astur). 2003;14(6):526-8.

5. Protocolos de neurocirurgia do Hospital de Pronto-Socorro João XXIII. Belo Horizonte: Hospital de Pronto-Socorro João XXIII; 2010.

6. Brain Trauma Foundation Guidelines. Disponível em: https:// www.braintrauma.org.

7. Jennett B, Bond M. Assessment of outcome after severe brain damage. Lancet. 1975;1(7905):480-4.

8. Seelig JM, Becker DP, Miller JD, Greenberg RP, Ward JD, Choi SC. Traumatic acute subdural hematoma: major mortality reduction in comatose patients treated within four hours. New Engl J Med. 1981;304:1511-8.

9. Ashkenazi E, Carmem M, Pasternak D, Israel Z, Beni L, Roneranz S. Conservative treatment of a traumatic subdural hematoma of the posterior fossa in a child: case report. $J$ Trauma. 1994;36(3):406-7

10. Motohashi O, Kameyama M, Shimosegawa Y, Fujimori K, Sugai K Onuma K. J Neurotrauma. 2002:19(8):993-8.

\footnotetext{
Endereço para correspondência

Leonardo Barnsley Araújo

Rua Dr. Juvenal dos Santos, 165, ap. 501

30380530 - Belo Horizonte, MG

Telefone: (31) 9132-2096

E-mail: lleobarnsley993@hotmail.com
} 